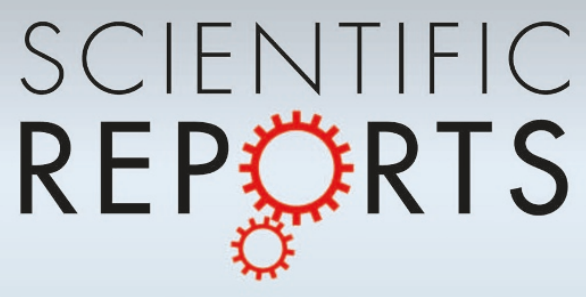

OPEN

SUBJECT AREAS:

COMPLEX NETWORKS

PHASE TRANSITIONS AND

CRITICAL PHENOMENA

NONLINEAR PHENOMENA

INFECTIOUS DISEASES

Received

3 February 2014

Accepted

13 June 2014

Published

16 July 2014

Correspondence and requests for materials should be addressed to G.T. (gouhei@sat.t.utokyo.ac.jp)

\section{Random and Targeted Interventions for Epidemic Control in Metapopulation Models}

\author{
Gouhei Tanaka',2, Chiyori Urabe ${ }^{2} \&$ Kazuyuki Aihara' ${ }^{1,2}$
}

${ }^{1}$ Graduate School of Engineering, The University of Tokyo, Tokyo 1 13-8656, Japan, ${ }^{2}$ Institute of Industrial Science, The University of Tokyo, Tokyo 153-8505, Japan.

In general, different countries and communities respond to epidemics in accordance with their own control plans and protocols. However, owing to global human migration and mobility, strategic planning for epidemic control measures through the collaboration of relevant public health administrations is gaining importance for mitigating and containing large-scale epidemics. Here, we present a framework to evaluate the effectiveness of random (non-strategic) and targeted (strategic) epidemic interventions for spatially separated patches in metapopulation models. For a random intervention, we analytically derive the critical fraction of patches that receive epidemic interventions, above which epidemics are successfully contained. The analysis shows that the heterogeneity of patch connectivity makes it difficult to contain epidemics under the random intervention. We demonstrate that, particularly in such heterogeneously connected networks, targeted interventions are considerably effective compared to the random intervention. Our framework is useful for identifying the target areas where epidemic control measures should be focused.

M odern transportation and traffic networks have enabled people to travel more frequently and globally than before, and therefore, epidemic outbreaks in local regions can potentially threaten international public health. The diversity and complexity of human mobility patterns complicate the planning of effective control measures against global epidemics at a local level. Therefore, epidemic control planning through international and inter-community cooperation is increasingly gaining importance to mitigate the threat of public health emergency caused by global epidemics ${ }^{1-3}$. The challenges to improve the detection and containment of epidemics with international importance have been addressed in international surveillance and response programs ${ }^{4}$, such as Global Outbreak Alert and Response Network (GOARN) ${ }^{5}$ supported by the World Health Organization (WHO). The collaboration networks of public health administrations and research institutes are expected to contribute to the planning of strategies and policies for better epidemic control measures. An assessment of the impact of potential control strategies is a critical issue for policymakers to implement them.

A metapopulation model is a useful mathematical framework to understand the dynamics of epidemic spreading in spatially separated patches, corresponding to countries, cities, or local communities, which are connected by pathways for human migration and population mobility ${ }^{6-9}$. Data analyses revealed the heterogeneous structures of transportation networks such as airline networks ${ }^{10-12}$, railway networks $s^{13,14}$, and commuting networks $s^{15,16}$, and therefore, metapopulation models with complex patch connectivity have been intensively studied ${ }^{15-29}$. A major concern in these studies has been the global epidemic threshold, which determines if a global epidemic occurs or not when a small number of infected individuals invade a local patch ${ }^{16,19,20,23-25}$. The metapopulation framework has also been widely employed to investigate the efficacy of containment measures such as travel restrictions ${ }^{19,21,28,29}$, vaccinations ${ }^{17}$, and therapeutic use of antiviral drugs ${ }^{21}$. Colizza $e t$ al.$^{21}$ demonstrated that the redistribution of the stockpiles of antiviral drugs between prepared and unprepared countries is effective under limited antiviral supplies. However, such a cooperative strategy for controlling the infection of subpopulations in local patches has yet to be fully examined.

Here, we present a metapopulation framework to evaluate the effectiveness of strategic interventions for epidemic control as compared to a non-strategic intervention. We consider a public health intervention that significantly reduces the infection risk of a subpopulation in a local patch through various countermeasures, e.g., the surveillance of cases and health indicators, delivery of vaccines, distribution of antiviral drugs, and promotion of hand hygiene. Public health interventions are provided to a fraction $p$ of the patches, which we refer to as the low-risk patches ${ }^{30}$. We assume that the infection rate in the low-risk patches is lower than that in the other patches 
without interventions. An increase in the ratio $p$ of the low-risk patches generally leads to a decrease in the prevalence of infection. Under certain conditions, there exists a critical ratio $p_{c}$ of the low-risk patches, above which the containment of epidemics is successful. Because the prevalence can differ depending on how to choose the low-risk patches, the critical ratio $p_{c}$ can also depend on the order in which low-risk patches are chosen as $p$ is increased. If we appropriately select the low-risk patches, then it would be possible to decrease the critical ratio $p_{c}$, implying a reduction in the total cost of public health interventions targeted at the local patches. Thus, we use the critical ratio $p_{c}$ as a measure for evaluating the effectiveness of public health interventions. First, we analytically derive the critical ratio for a random (non-strategic) intervention where low-risk patches are randomly chosen from the whole patches. Second, we numerically examine the effect of strategic interventions where the low-risk patches are chosen based on available information. The results show that strategic interventions are highly effective compared to the random intervention in metapopulation models with heterogeneous connectivity. Finally, numerical simulations performed using a Japanese airline network demonstrate that our framework is useful to specify the best intervention strategy and its target areas.

Random and targeted interventions for controlling epidemics have been widely argued based on both contact networks of individuals and the metapopulation models with networked patches. However, the targeted immunization in the contact networks corresponds to removal of the static nodes (i.e. the individuals) which do not have dynamics ${ }^{31-35}$. Therefore, the results on the immunization strategies based on the contact networks cannot straightforwardly apply to the intervention strategies at the metapopulation level. The targeted intervention in our metapopulation framework changes the transmission rate in the local patches. Such a model framework is not found in most of the relevant studies on metapopulation models where the transmission rates (or the local basic reproductive number) are assumed to be identical. As far as we know, there is only one study taking into account patch differences in the local transmission rates, where the epidemic threshold is analyzed with respect to the mobility parameter ${ }^{30}$. The threshold with respect to the fraction of low-risk patches, which we focus on in the present study, gives a new perspective. This viewpoint enables to examine the strategies for epidemic control based on local interventions, in a framework different from the previous ones. Although we employ a deterministic model without stochastic effects and assume a simple mobility pattern and simple infection dynamics in order to highlight the usefulness of our framework and analysis, the method is applicable to more complicated metapopulation models incorporating stochastic fluctuations, realistic human mobility patterns ${ }^{16,27}$, and realistic human behavior $^{28}$.

\section{Results}

Metapopulation models with epidemic intervention. We consider epidemic spreading in a population of individuals, separated into subpopulations in spatially discrete patches connected by migration pathways. The model is represented by a metapopulation model consisting of $N$ patches, in each of which susceptible and infected individuals are homogeneously mixed ${ }^{19,24,25}$. The epidemic dynamics in each local patch is described using a susceptibleinfectious-susceptible (SIS) compartment $\operatorname{model}^{36,37}$ : In patch $i$ for $i=1, \ldots, N$, a susceptible individual interacting with an infected individual turns into an infected one at a transmission rate $\beta_{i}$ and an infected individual recovers to be susceptible at a recovery rate $\mu_{i}$. Note that, although we use the SIS model for simplicity in this paper, our framework is also applicable to other compartment models of epidemic spreading. Here we assume non-limited transmission for simplicity, in which the total number of contacts of the individuals within a patch increases with an increase in the population density of the patch ${ }^{19,24,25}$ (see Supplementary Information for the theoretical analysis of the case with limited transmission, in which the contact rate is constant independently of the population density). If patch $i$ is isolated, the epidemic threshold is given by the local basic reproduction number defined as $R_{0}^{(i)} \equiv \beta_{i} / \mu_{i}$ for the SIS model. In metapopulation models, however, the global invasion threshold depends on the other factors such as population density, patch connectivity, and mobility rates ${ }^{19,24,25}$. The total density of the individuals is represented by $\rho$. The connectivity of the patches is characterized by the degree $k_{i}$ for $i=1, \ldots, N$, which represents the number of migration pathways for patch $i$. The mobility rates of susceptible and infected individuals are represented by $D_{\mathrm{S}}$ and $D_{\mathrm{I}}$, respectively. Each individual in a patch randomly chooses one of the pathways departing from the patch and migrates to another patch with the mobility rate. The dynamics of epidemic spreading in the metapopulation model is divided into two processes: transmission or recovery within local patches (the reaction process) and migration of individuals between patches (the diffusion process) ${ }^{19,24,25}$. If the reaction and diffusion processes occur simultaneously, then a suitable continuous-time formulation of the metapopulation dynamics can be derived ${ }^{24,25}$. We employ this continuous-time SIS metapopulation model.

We incorporate public health interventions that lower the infection risk in a local patch into the metapopulation model. The whole patches are divided into low-risk patches with interventions and high-risk patches without interventions. For simplicity, we assume that the recovery rate is common for all patches, i.e., $\mu_{i}=\mu$, and the transmission rates are different between the two types of patches, i.e., $\beta_{i}=\beta_{\mathrm{L}}<\mu$ for the low-risk patches and $\beta_{i}=\beta_{\mathrm{H}}>\mu$ for the highrisk patches. The local basic reproduction number for the low-risk patch is given by $R_{0}^{(\mathrm{L})}=\beta_{\mathrm{L}} / \mu<1$ and that for the high-risk patch by $R_{0}^{(\mathrm{H})}=\beta_{\mathrm{H}} / \mu>1$. Therefore, a disease-free state without infected individuals is stable in an isolated low-risk patch, whereas an endemic steady state with a certain number of infected individuals is stable in an isolated high-risk patch. In the metapopulation model where the low-risk and high-risk patches coexist, the steady state depends on the ratio between the two types of patches and their configurations. Figure 1 schematically illustrates the metapopulation model that consists of low-risk and high-risk patches. The ratio of the low-risk patches to the whole patches is represented by $p$ with $0 \leq p$ $\leq 1$. A large value of $p$ indicates that the public health interventions are more costly in total. Let us suppose that the steady state is an endemic state for $p=0$ and a disease-free state for $p=1$. In such a case, it is expected that there exists a critical value $p=p_{c}$, where a transition between the two states occurs. In this study, the critical low-risk patch ratio $p_{c}$ is used as a measure for evaluating the effectiveness of the interventions for epidemic control.

Critical low-risk patch ratio for random intervention. We investigate a random intervention where low-risk patches are randomly chosen from the whole patches. For the SIS metatpopulation model, the epidemic size is evaluated by the prevalence of infection $\rho_{\mathrm{I}} / \rho$, where $\rho_{\mathrm{I}}$ denotes the average density of infected individuals (see Methods section for the numerical simulation method). Figure 2 demonstrates that the prevalence declines with an increase in the low-risk patch ratio $p$ in both homogeneous and heterogeneous metapopulations with the same mean degree. The homogeneous networks are given by the Erdős-Rényi random graphs ${ }^{38}$ and the heterogeneous networks are by the Barabási-Albert scale-free networks that are generated using a preferential attachment rule ${ }^{39}$. In the homogeneous networks, the decline rate of the prevalence of infection is higher than that in the heterogeneous ones. We can clearly observe an existence of the critical low-risk patch ratio $p_{c}$, above which the prevalence is zero in the homogeneous networks. In contrast, the prevalence does not appear to fall to zero even for a sufficiently large $p$ in the heterogeneous networks. The result suggests that the random intervention is not effective for the 


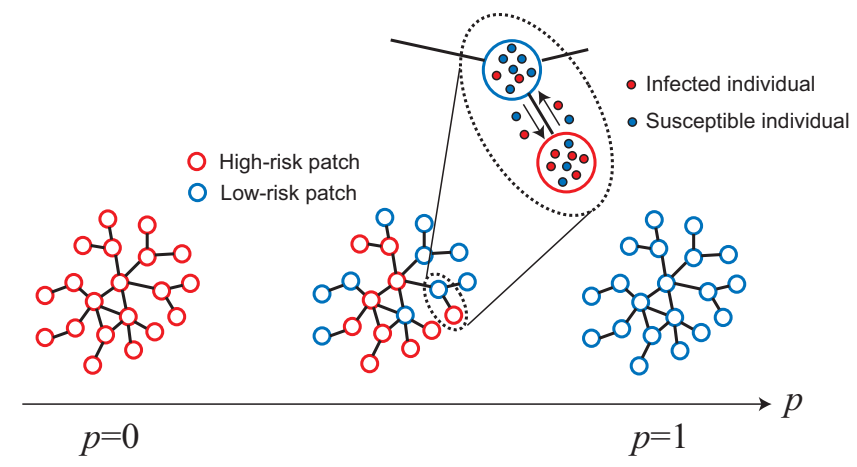

Figure 1 Metapopulation model with interventions for epidemic control in local patches. Schematic illustration of a metapopulation model consisting of high-risk patches without interventions (red open circles) and low-risk patches with interventions (blue open circles). The individuals in the patches are either susceptible (blue filled circles) or infected (red filled circles). In each patch, the disease transmission and recovery of individuals occur according to the SIS compartment model. The local basic reproduction number in the high-risk patches is given by $R_{0}^{(\mathrm{H})}=\beta_{\mathrm{H}} / \mu>1$ and that in the low-risk patches by $R_{0}^{(\mathrm{L})}=\beta_{\mathrm{L}} / \mu<1$. The parameter $p$ represents the ratio of the low-risk patches to the whole patches, corresponding to the total size of public health interventions.

containment of epidemics if the patch connectivity is heterogeneous as in real transportation networks.

For the random intervention, we analytically derive the critical low-risk patch ratio $p_{c}$ using heterogeneous mean-field approximation $^{22,40-42}$. For the mean-field approximation to work effectively, we assume uncorrelated networks with a sufficiently large number $N$ of the patches. When the mobility rates are positive, i.e., $D_{\mathrm{S}}>0$ and $D_{\mathrm{I}}>0$, we obtain the critical low-risk patch ratio $p_{c}$ as follows (see Methods section and Supplementary Information for details):

$$
p_{c}=\frac{1-F\left(\beta_{\mathrm{H}}\right)}{F\left(\beta_{\mathrm{L}}\right)-F\left(\beta_{\mathrm{H}}\right)},
$$

with

$$
F(\beta) \equiv \sum_{k} P(k) \frac{D_{\mathrm{I}} k /\langle k\rangle}{\mu+D_{\mathrm{I}}-\rho \beta k /\langle k\rangle},
$$

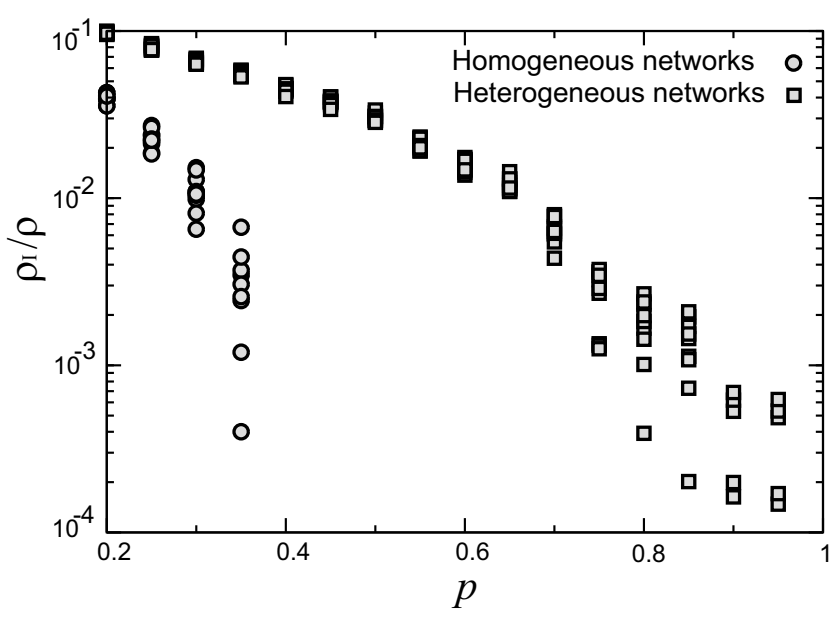

Figure $2 \mid$ Transition between endemic and disease-free states for random intervention. The prevalence of infected individuals, $\rho_{\mathrm{I}} / \rho$, is plotted against the low-risk patch ratio $p$. The circles and squares indicate the results for homogeneous and heterogeneous networks with $N=1000$ and $\langle k\rangle \approx 10$, respectively. For each network type, the results for ten different network realizations are superimposed. The parameter values are set at $\rho=1, D_{\mathrm{S}}=0.1, D_{\mathrm{I}}=5, \mu=1, \beta_{\mathrm{H}}=1.2$, and $\beta_{\mathrm{L}}=0.2$. where $P(k)$ represents the degree distribution and $\langle k\rangle \equiv \sum_{i=1}^{N} k_{i} / N$ represents the mean degree. A necessary condition for Eq. (1) is given by the inequality

$$
\rho<\frac{\langle k\rangle}{k_{\max }} \frac{\mu+D_{\mathrm{I}}}{\beta_{\mathrm{H}}},
$$

where $k_{\max }$ represents the maximum degree in the network. If inequality (3) does not hold, there exists a high-risk patch with degree $k$ for which the denominator of the right-hand side of equation (2) vanishes. In such a case, $F\left(\beta_{\mathrm{H}}\right)$ diverges, and thereby, $p_{c}$ goes to 1 . The condition given by inequality (3) is equivalent to an exclusion of the possibility that the disease-free state is unstable for any $p$ in the range of $0 \leq p<1$. See Supplementary Information for details and the results for the case with limited transmission.

Figure 3 shows the phase diagrams in the $\left(D_{\mathrm{I}}, p\right)$-parameter plane. The colors indicate the prevalence of infection in the steady state, separating the endemic (red) and disease-free (blue) states. The boundary between the two states corresponds to the critical low-risk patch ratio $p_{c}$. The theoretical values of $p_{c}$ obtained from equation (1) are in good agreement with the boundary in both the homogeneous and heterogeneous networks.

Strategic interventions. Next, let us examine strategic interventions. The critical low-risk patch ratio $p_{c}$ depends on the procedure of selecting the low-risk patches with an increase in $p$. We consider three different types of intervention strategies that can reduce the value of $p_{c}$ as compared to the random intervention. $A n$ neighborhood intervention is inspired by an immunization strategy, called acquaintance immunization, on complex human contact networks ${ }^{35}$. In this strategy, all patches are assumed as high-risk patches initially at $p=0$. Then, a patch is randomly chosen from the whole patches and one of its neighboring high-risk patches is changed to a low-risk patch at random. This procedure is repeated until the number of the low-risk patches reaches $p N$. A highly connected patch tends to be chosen as a low-risk patch by this procedure. The advantage of this intervention is that it only requires information about local patch connectivity. A targeted intervention is conducted based on centrality, which indicates the relative importance of the patch node from the viewpoint of network connectivity ${ }^{43,44}$. We only show the results for the usage of degree centrality, because the same results were obtained for the usage of closeness, betweenness, and eigenvector centralities. In this strategy, a high-risk patch with a higher centrality is more likely to be chosen as a low-risk patch. The targeted intervention requires information about global patch connectivity to compute the centrality values of the patches ${ }^{33}$. An adaptive intervention is introduced to test an adhoc intuitive manner in which interventions are targeted at unsafe patches with high morbidity rates. In this strategy, a high-risk patch with a high morbidity rate is preferentially chosen as a low-risk patch. Because morbidity rates in local patches vary with time, the choice of low-risk patches is adaptively updated at a fixed time interval $T$.

Effectiveness of strategic interventions. We numerically examine the effectiveness of the neighborhood, targeted, and adaptive interventions in comparison to the baseline case with the random intervention. The best strategy, which enables us to contain epidemics with the smallest number of low-risk patches, can be identified in our simulations. The simulations were performed for different networks: homogeneous networks ${ }^{38}$, heterogeneous networks ${ }^{39}$, and an US airline network ${ }^{19}$. We introduce a parameter $\Delta \beta$ to simultaneously change the transmission rates in both the highrisk and low-risk patches; they are given by $\beta_{\mathrm{H}}=1+\Delta \beta$ and $\beta_{\mathrm{L}}=$ $\Delta \beta$, respectively. As $\Delta \beta$ increases, the transmission rate increases in all patches, and thereby make it difficult to contain an epidemic.

Figure 4 shows the phase diagrams separating the endemic and disease-free states in the parameter plane of $\Delta \beta$ and $p$ for the homo- 

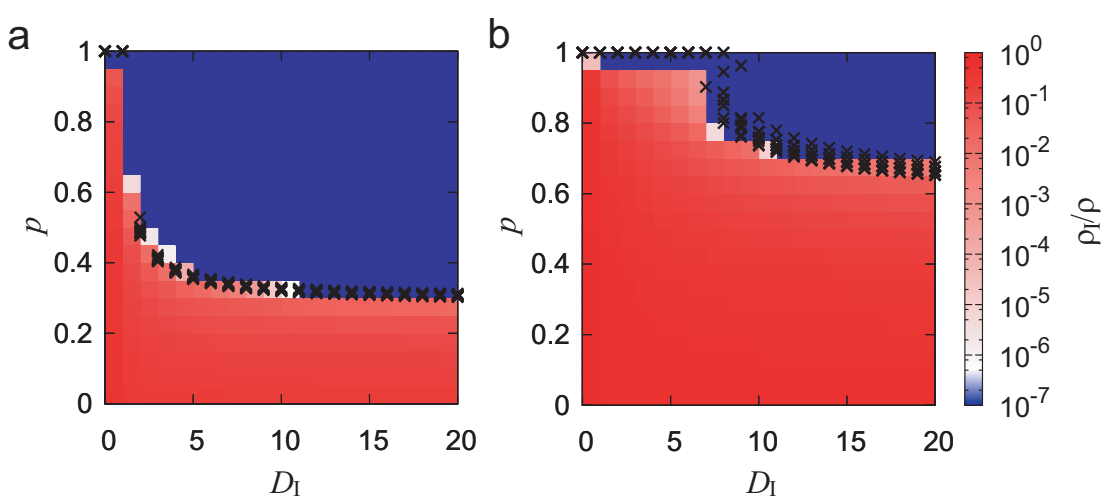

Figure 3 Validation of the analytical expression of the critical low-risk patch ratio for random intervention. Phase diagrams on the $\left(D_{\mathrm{I}}, p\right)$ parameter plane, where $D_{\mathrm{I}}$ is the mobility rate of the infected individuals and $p$ is the low-risk patch ratio. The color bar indicates the numerically obtained values of the prevalence $\rho_{\mathrm{I}} / \rho$, averaged over ten different network realizations. The red and blue regions correspond to the endemic and disease-free states, respectively. The boundary between the two states corresponds to the critical low-risk patch ratio $p_{c}$. The crosses indicate the theoretical values of $p_{c}$ obtained from equation (1) for the same ten network realizations. The parameter values are set at $\rho=1, D_{\mathrm{S}}=0.1, \mu=1, \beta_{\mathrm{H}}=1.2$, and $\beta_{\mathrm{L}}=0.2$. (a) Homogeneous networks with $N=1000$ and $\langle k\rangle \approx 10$. (b) Heterogeneous networks with $N=1000$ and $\langle k\rangle \approx 30$.

geneous networks. It is reasonable that more interventions are necessary to contain the epidemic for a larger value of $\Delta \beta$ in all the cases. Figure 4a shows the result for the random intervention, which is the baseline for comparing all intervention strategies. The result for the neighborhood intervention is almost similar to that for the random intervention, as shown in Fig. $4 \mathrm{~b}$. The targeted intervention based on the degree centrality is slightly better than the random intervention, as shown in Fig. 4c. Figures $4 \mathrm{~d}, 4 \mathrm{e}$, and $4 \mathrm{f}$ present results for the adaptive interventions with different update intervals $T=10,50$, and 100 , respectively. Overall, strategic interventions are not very beneficial for homogeneous networks with a narrow degree distribution.

Figure 5 shows the phase diagrams for heterogeneous networks with broad degree distributions. As shown in Fig. 5a, the random intervention fails to contain the epidemic unless the interventions are given to nearly all patches. The neighborhood intervention is much better than the random intervention as shown in Fig. 5b. This is because high-degree patches containing a large population of individuals tend to be chosen as low-risk patches in this strategy. Figure $5 \mathrm{c}$ shows that the targeted intervention based on the degree centrality is highly effective. The targeted interventions based on closeness, betweenness, and eigenvector centralities produced almost the same results, because all centrality measures are positively correlated to each other for the heterogeneous networks. Figures $5 \mathrm{~d}, 5 \mathrm{e}$, and $5 \mathrm{f}$ show the results for the adpaptive interventions with different update intervals $T=10,50$, and 100 , respectively. These interventions are better than the neighborhood intervention, but not than the targeted intervention. In particular, the frequent update of low-risk patches in the case of $T=10$ appears to be the best. The time evolutions of the average density of infected individuals exhibit oscillations due to the switching of dynamics, resulting from the update of low-risk patches (see Supplementary Fig. S3). The interplay between the continuous dynamics of epidemic spreading and the discrete switching of the low-risk patches causes a non-monotonic increase in the critical low-risk patch ratio with an increase in $\Delta \beta$ as found in Figs. 5e and 5f. We can conclude that all the above mentioned strategic interventions are highly effective compared to the random intervention, and the best strategy is the targeted intervention in the heterogeneous networks.

To confirm the efficacy of strategic interventions in real heterogeneous networks, we performed similar numerical experiments using the airline transportation networks in the United States ${ }^{19}$. The total number of airports is 500 and the total number of flight routes is 2980 . The distribution of the number of flight routes shows a power-law decay, implying that the network has a scale-free property. Figure 6 shows the results for the random and strategic interventions. The strategic interventions are considerably better than the random intervention, as observed in the previous results for the model networks with the scale-free property. However, in the US airline network, the adaptive interventions are less effective than the neighborhood intervention.

Identification of target areas of intervention. Finally, we demonstrate that our framework is useful to identify the target areas for public health intervention using real human mobility patterns on the domestic airline network in Japan. From the time tables of domestic flights in All Nippon Airways (http://www.ana.co. jp) and Japan Airlines (http://www.jal.co.jp), we obtained the data about the total connectivity of the airline networks. Figure $7 \mathrm{a}$ illustrates the map of the airports in Japan. Further, we estimated the weights of the connections from the number of passengers traveling through each route in one day. The number of airports is $N=74$ and the total number of routes is 190 . The degree distribution is shown in Fig. 7b, approximately following a power law. The mean, maximum, and minimum degrees are given by $\langle k\rangle \approx 5.1, k_{\max }=50$ (corresponding to the Tokyo-Haneda airport), and $k_{\min }=1$, respectively. Both the connection and weight matrices are symmetric. Because the weights are different between the flight routes as shown in Fig. 7b, we employ the metapopulation model with heterogeneous diffusion (see Methods section). The patches are assumed to correspond to the local cities around the airports. The initial density of individuals in each patch is set to be proportional to the total number of passengers at the corresponding airport.

Figure $7 \mathrm{~d}$ shows the prevalence of infection with the variation of the low-risk patch ratio $p$ under the random and strategic interventions. The targeted intervention based on the degree centrality is the most successful strategy for the containment of epidemics by controlling only $5 \%$ of the patches, i.e., Tokyo (Haneda), Okinawa (Naha), Osaka (Itami), and Sapporo (New-Chitose). The second option would be the neighborhood intervention. The value of $p_{c}$ ranged between $8 \%$ and $16 \%$ for five simulations of this strategy. The adaptive intervention is not considerably different from the random intervention. Thus, our framework is useful to specify the best intervention strategy and the target areas where the control measures should be preferentially concentrated. If available resources and costs for epidemic control are limited, the best option can be found by comparing the prevalence for different interventions at a fixed value $p$. 
a

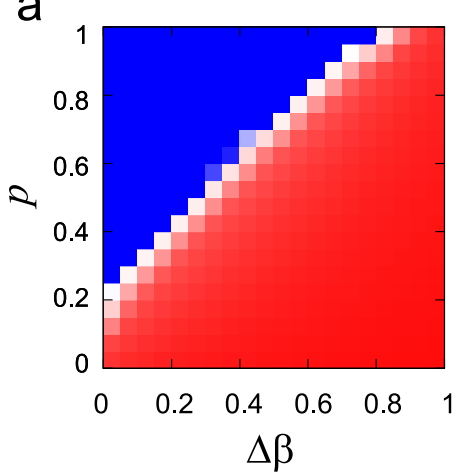

d

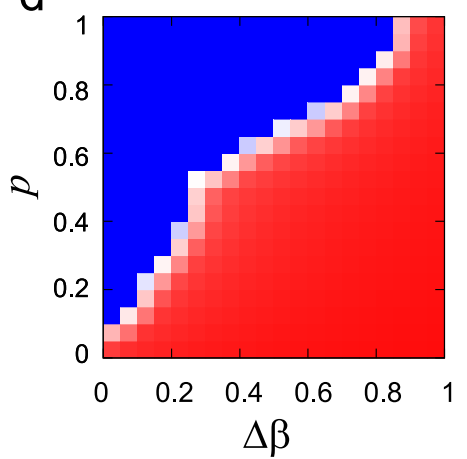

b

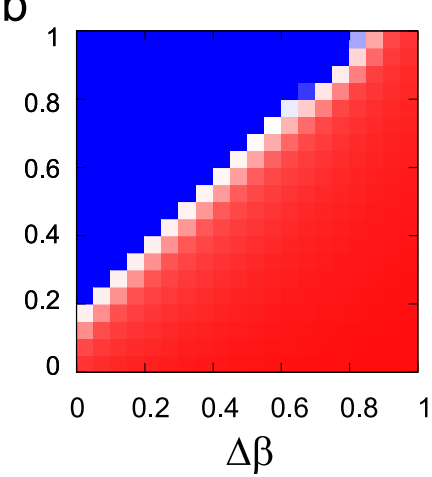

e

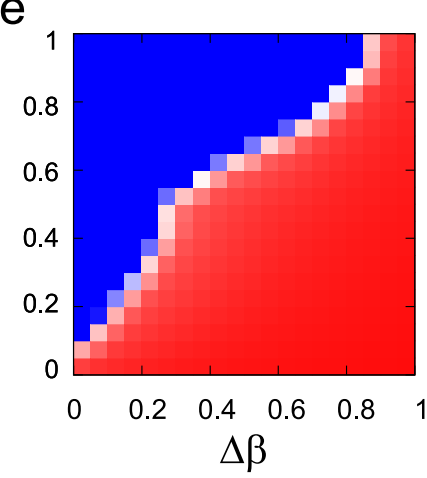

C

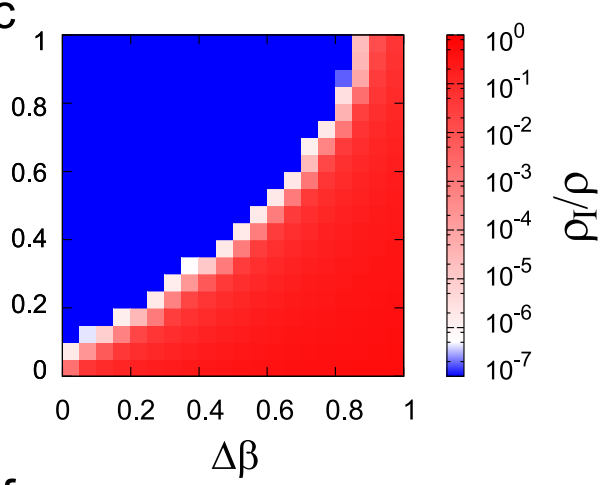

f

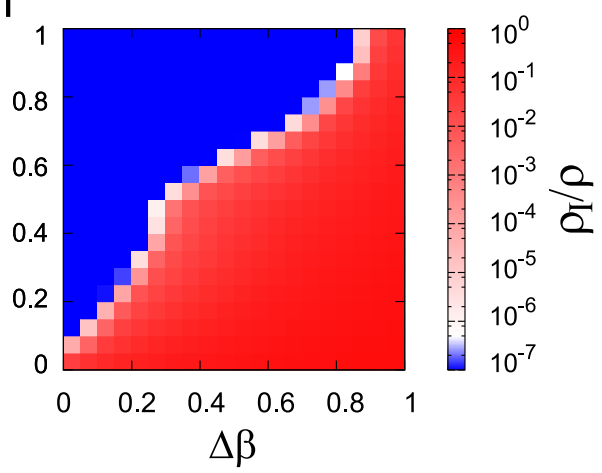

Figure 4 | Effects of epidemic interventions in metapopulation models with homogeneous patch connectivity. Each panel shows the phase diagram for homogeneous networks in the $(\Delta \beta, p)$ parameter plane, where $\Delta \beta$ controls the transmission rates given by $\beta_{\mathrm{H}}=1+\Delta \beta$ and $\beta_{\mathrm{L}}=\Delta \beta$. The color bar indicates the numerically obtained values of the prevalence $\rho_{\mathrm{I}} / \rho$, averaged over ten different network realizations with $N=1000$ and $\langle k\rangle \approx 10$. The red and blue regions correspond to the endemic and disease-free states, respectively. The parameter values are set at $\rho=1, D_{\mathrm{S}}=0.1$, and $D_{\mathrm{I}}=5$. (a) Random intervention; (b) Neighborhood intervention; (c) Targeted intervention; (d) Adaptive intervention with $T=10$; (e) Adaptive intervention with $T=50$; and (f) Adaptive intervention with $T=100$.

\section{a}

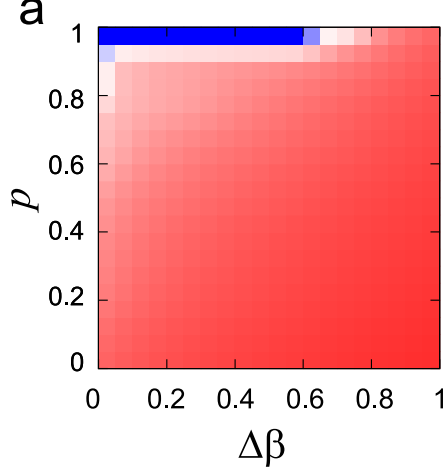

d

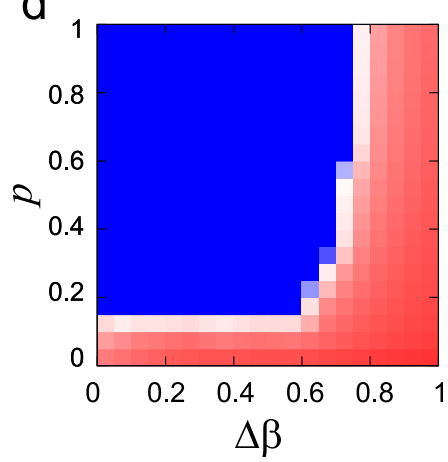

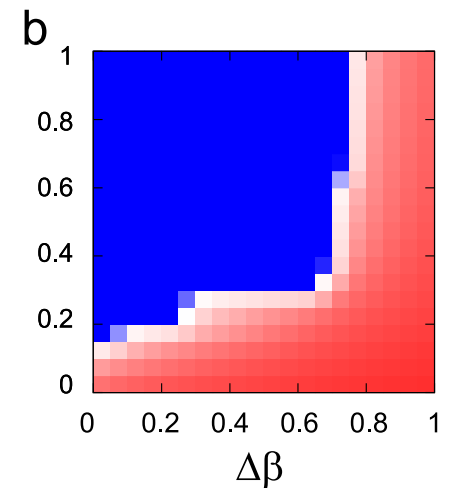

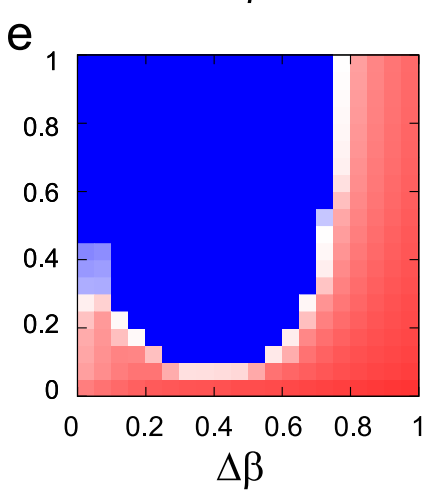

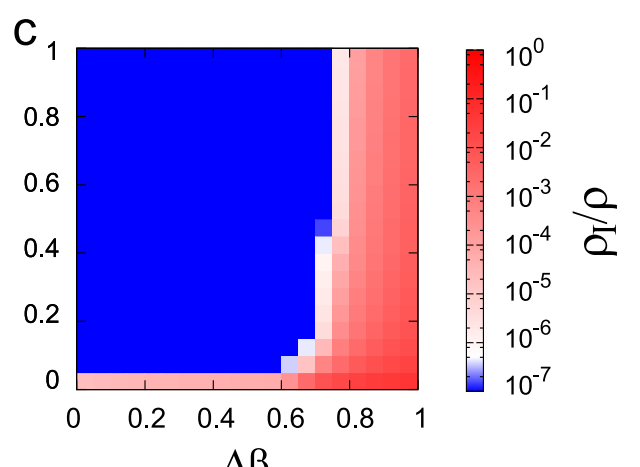

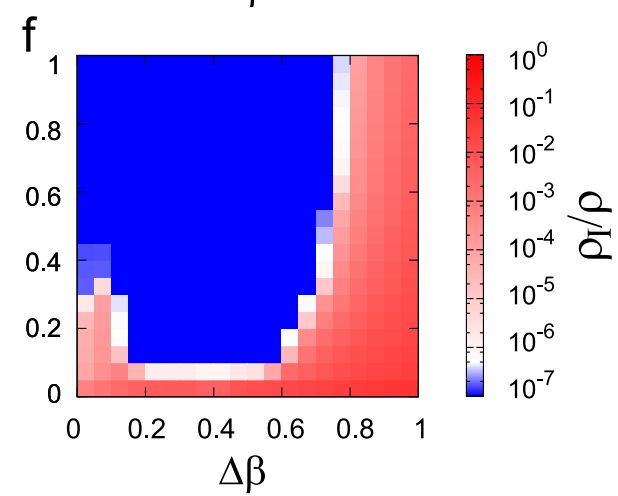

Figure $5 \mid$ Effects of epidemic interventions in metapopulation models with heterogeneous patch connectivity. The same as Fig. 4, but for heterogeneous networks with $N=1000$ and $\langle k\rangle \approx 30$. The parameter values are set at $\rho=0.4, \mu=1, D_{\mathrm{S}}=0.1$, and $D_{\mathrm{I}}=5$. 

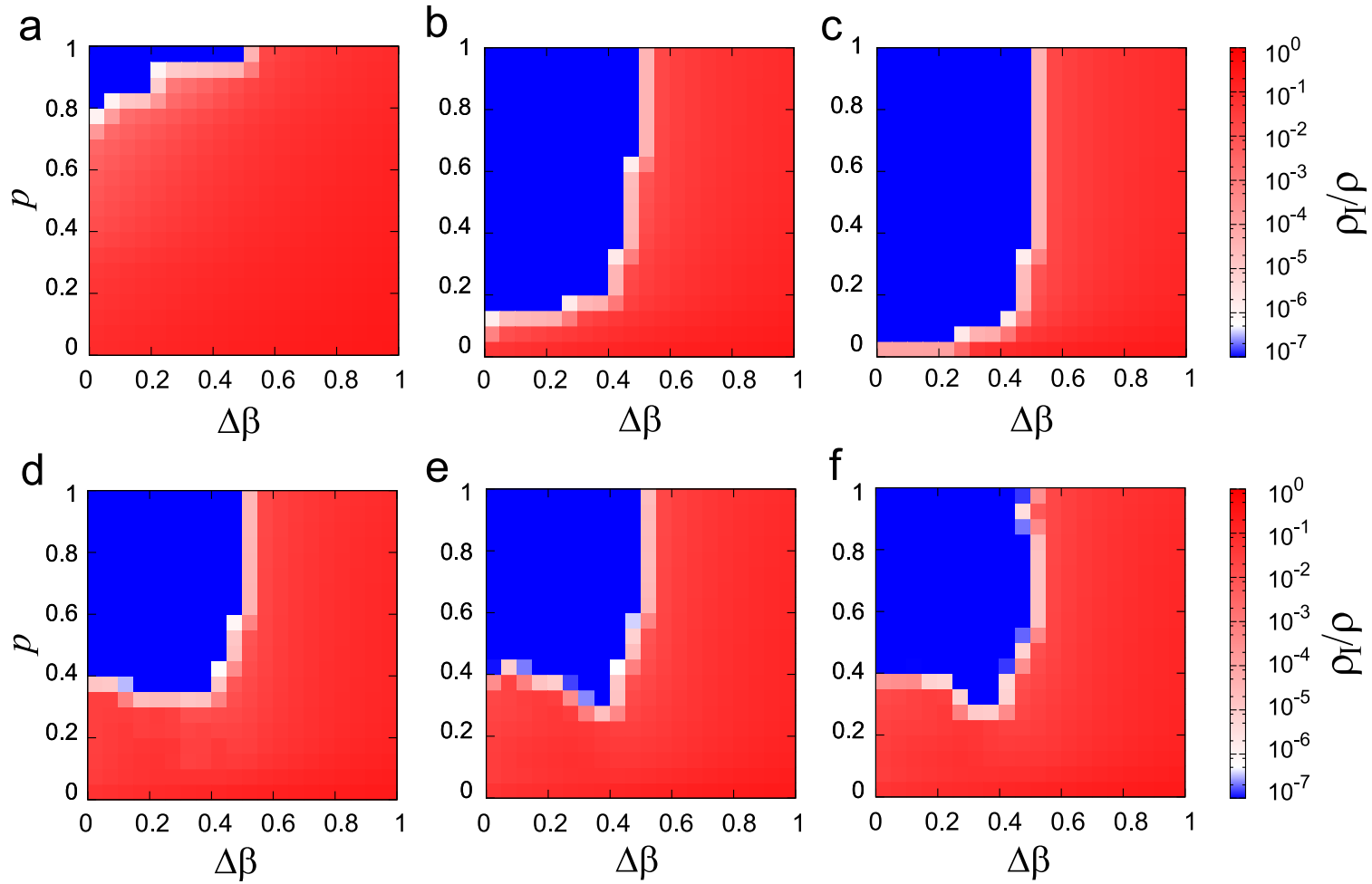

Figure 6 Effects of epidemic interventions in metapopulation models with US airline networks. The same as Figs. 4 and 5, but for US airline networks ${ }^{19}$ with $N=500$ and $\langle k\rangle \approx 6$. The parameter values are set at $\rho=1, \mu=1, D_{\mathrm{S}}=0.1$, and $D_{\mathrm{I}}=5$.
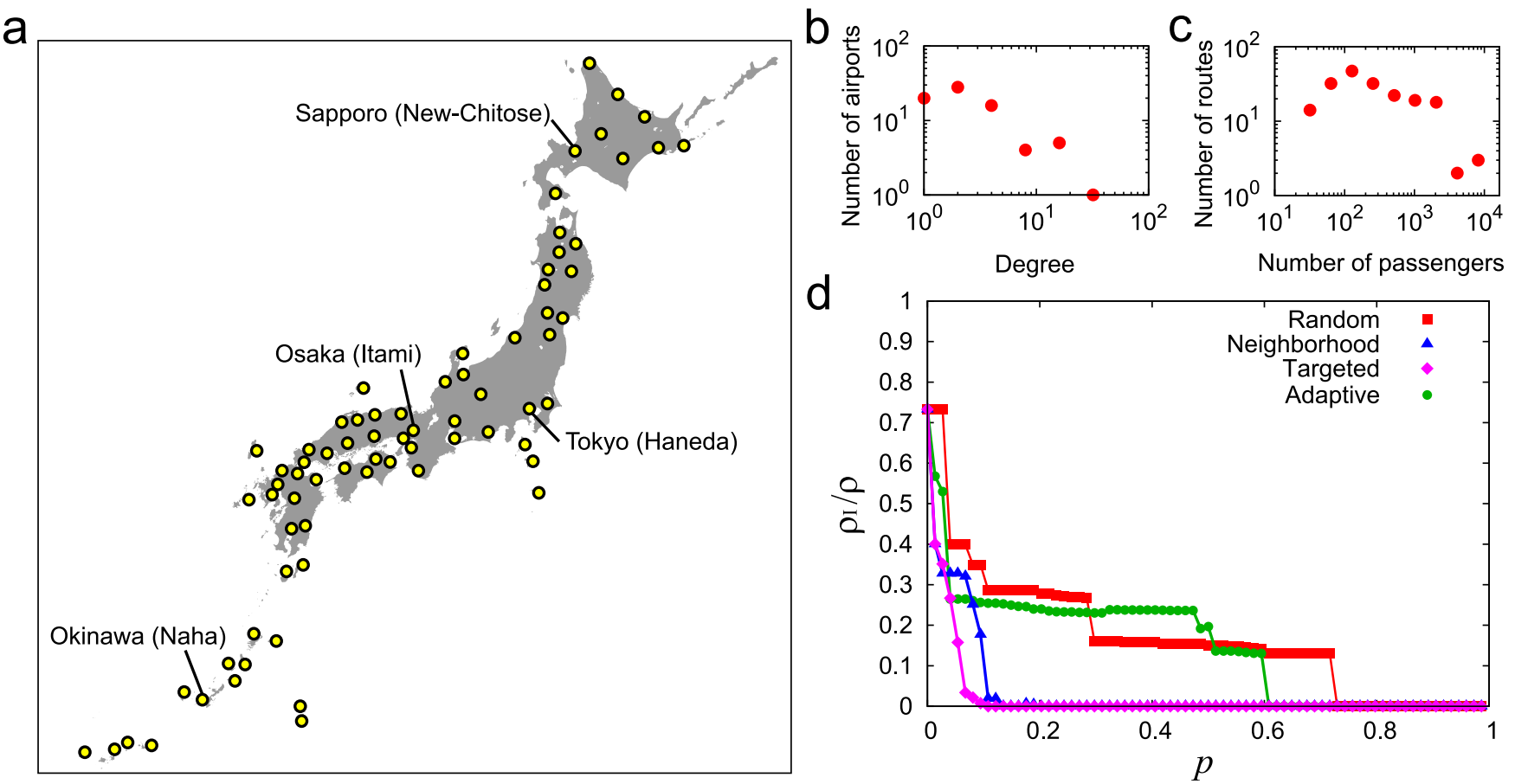

Figure 7 Epidemic interventions in metapopulation models with the Japanese airline network. The data of the Japanese airport network were obtained from the timetables of domestic flights of All Nippon Airway (http://www.ana.co.jp) and Japan Airline (http://www.jal.co.jp). The Japanese airport network consists of $N=74$ airports and 190 flight routes. The mean, maximum, and minimum degrees are given by $\langle k\rangle \approx 5.1, k_{\max }=50$ (corresponding to the Tokyo-Haneda airport), and $k_{\min }=1$, respectively. (a) The map of the airports in Japan, which was created with Adobe Illustrator; (b) The degree distribution of the Japanese airport network; (c) The distribution of the number of passengers per flight route; and (d) The prevalence $\rho_{\mathrm{I}} / \rho$ is plotted against the low-risk patch ratio $p$ for the random (squares), neighborhood (triangles), targeted (diamonds), and adaptive (circles) interventions. For the random and neighborhood interventions, the best result among five simulations was adopted. For adaptive intervention, the time interval for updating the low-risk patches is set at $T=10$. The other parameter values are set at $\rho=0.5, \mu=1, D_{\mathrm{S}}=D_{\mathrm{I}}=10, \beta_{\mathrm{H}}=2$, and $\beta_{\mathrm{L}}=0.1$. 


\section{Discussion}

We have presented a mathematical framework to evaluate the effectiveness of the random and targeted epidemic interventions in the SIS metapopulation models. First, we have obtained a theoretical result on the critical low-risk patch ratio for the random intervention and confirmed its validity by performing numerical simulations. We have shown that the heterogeneity of patch connectivity makes it difficult to contain epidemics. Then, we have compared the efficacy of intervention strategies using the result for the random intervention as a baseline. We have found that strategic interventions are considerably more effective than the random intervention in heterogeneously connected networks. Moreover, the targeted intervention based on the degree centrality has shown to be most effective compared to the neighborhood and adaptive interventions. This result suggests that epidemic control measures should be focused on the highly connected areas. In contrast, the adaptive intervention can be worse than the neighborhood intervention. Our investigation has highlighted the importance of strategic interventions for epidemic control in mitigating and containing global epidemics. The optimum strategy can be selected with the support of the mathematical framework presented in this work.

Our framework is applicable to more extended situations. The SIS model for disease transmission can be changed to other compartment models such as susceptible-infectious-recovered (SIR) and susceptible-exposed-infectious-recovered (SEIR) models. For these models, an appropriate measure for the epidemic size should be the cumulative number of infected individuals during the period of an outbreak, instead of the prevalence that is used for the SIS model. We have considered the two transmission rates in the local patches for simplicity. A full exploration for the impact of the combination of the transmission rates on epidemic spreading is an issue to be studied in the future. Further, a more heterogeneous distribution of the transmission rates in the patches is a realistic factor to be incorporated into the model. We have assumed a simple human mobility pattern (diffusion process) as a first step to test our framework. The human mobility pattern can be replaced by more realistic mobility patterns such as recurrent ${ }^{15}$, natural ${ }^{27}$, and safety-information-driven $^{29}$ types. Furthermore, it is possible to consider other intervention strategies which have not been tested in this work; the preferential order of intervention can be determined based on other information characterizing the patches, such as the local population density.

\section{Methods}

Continuous-time metapopulation model. A metapopulation model consisting of $N$ patches is considered. In each patch, the infection and recovery of individuals occur according to the susceptible-infectious-susceptible (SIS) model. In the continuoustime formulation for the progress of diseases on metapopulations, the equations for the dynamics in patch $i(1 \leq i \leq N)$ are described as follows ${ }^{24,25}$ :

$$
\begin{gathered}
\frac{\mathrm{d} \rho_{\mathrm{S}, i}}{\mathrm{~d} t}=-\beta_{i} \rho_{\mathrm{S}, i} \rho_{\mathrm{I}, i}+\mu_{i} \rho_{\mathrm{I}, i}-D_{\mathrm{S}} \rho_{\mathrm{S}, i}+D_{\mathrm{S}} \sum_{j=1}^{N} a_{j i} d_{j i} \rho_{\mathrm{S}, j}, \\
\frac{\mathrm{d} \rho_{\mathrm{I}, i}}{\mathrm{~d} t}=\beta_{i} \rho_{\mathrm{S}, i} \rho_{\mathrm{I}, i}-\mu_{i} \rho_{\mathrm{I}, i}-D_{\mathrm{I}} \rho_{\mathrm{I}, i}+D_{\mathrm{I}} \sum_{j=1}^{N} a_{j i} d_{j i} \rho_{\mathrm{I}, j},
\end{gathered}
$$

where $\rho_{\mathrm{S}, i}$ and $\rho_{\mathrm{I}, i}$ represent the numbers of susceptible and infected individuals in patch $i$, respectively. The epidemiological parameters, $\beta_{i}$ and $\mu_{i}$, denote the transmission and recovery rates in patch $i$, respectively. The term $\beta_{i} \rho_{\mathrm{s}, i} \rho_{\mathrm{I}, i}$ represents the nonlimited transmission, whereas the transmission term is given by $\beta_{i} \rho_{\mathrm{S}, i} \rho_{\mathrm{I}, i} /\left(\rho_{\mathrm{S}, i}\right.$ $\left.+\rho_{\mathrm{I}, i}\right)$ for the limited transmission ${ }^{19,24,25}$. The recovery rate is assumed to be the same in all patches, i.e., $\mu_{i}=\mu$. The transmission rate is set at $\beta_{i}=\beta_{\mathrm{L}}<\mu$ for low-risk patches and at $\beta_{i}=\beta_{\mathrm{H}}>\mu$ for high-risk patches. The ratio of low-risk patches to the whole patches is denoted by $p$. The parameters $D_{\mathrm{S}}$ and $D_{\mathrm{I}}$ denote the mobility rates of susceptible and infected individuals, respectively. The bidirectional connectivity of the patches is characterized by the $N \times N$ adjacency matrix $A=\left(a_{i j}\right): a_{i j}=a_{j i}=1$ if patch $i$ and patch $j$ are connected; otherwise $a_{i j}=a_{j i}=0$. The degree of patch $i$ is given by $k_{i}=\sum_{j=1}^{N} a_{i j}$. When the diffusion is heterogeneous, connection weights are characterized by the $N \times N$ weight matrix $W=\left(w_{i j}\right)$. The weight $w_{i j}\left(=w_{j i}\right)$ is proportional to the number of passengers traveling between patch $i$ and patch $j$. The diffusion rate is given by (i) $d_{i j}=1 / k_{i}$ for the homogeneous diffusion and (ii) $d_{i j}=w_{i j} /\left(\sum_{i=1}^{N} w_{i j}\right)$ for the heterogeneous diffusion ${ }^{23}$.

Numerical simulation method. We simulated equations (4)-(5) with the fourthorder Runge-Kutta method with time step 0.01 for $t \in\left[0, t_{\max }\right]$, where $t_{\max }=2000$. The total density of the individuals, $\rho=\sum_{i=1}^{N}\left(\rho_{\mathrm{S}, i}(t)+\rho_{\mathrm{I}, i}(t)\right) / N$, remains constant over time $t$. The epidemic size was measured by the prevalence of infection $\rho_{\mathrm{I}} / \rho$, where $\rho_{\mathrm{I}}=\sum_{i=1}^{N} \rho_{\mathrm{I}, i}\left(t_{\max }\right) / N$ is the average density of infected individuals for the random, neighborhood, and targeted interventions. For adaptive intervention producing oscillatory behavior of $\rho_{\mathrm{I}}(t)$ with period $2 T$, the prevalence was measured by $\rho_{\mathrm{I}}=(1 / 2 T) \sum_{t=t_{\max }}^{t=\max _{2}+2 T} \sum_{i=1}^{N} \rho_{\mathrm{I}, i}(t) / N$.

Mean-field approximation. As the low-risk patch ratio $p$ increases from a sufficiently small value, the initially unstable disease-free equilibrium is stabilized at a critical value $p=p_{c}$. Using the heterogeneous mean-field approximation ${ }^{22,40-42}$, we analytically derive the critical low-risk patch ratio $p_{c}$ for the metapopulation model (4)-(5) with uncorrelated networks and the homogeneous diffusion, i.e., $d_{i j}=1 / k_{i}$. We consider the case with positive mobility rates $D_{\mathrm{S}}>0$ and $D_{\mathrm{I}}>0$ (see Supplementary Information for the other cases). We assume that inequality (3) is satisfied to exclude the case in which the disease-free equilibrium is unstable for any $p$ in the range of $0 \leq p<1$. Given the average density of infected individuals in the steady state, $\bar{\rho}_{\mathrm{I}}=\sum_{i=1}^{N} \rho_{\mathrm{I}, i} / N$, we can rewrite the model (4)-(5) as follows:

$$
\begin{gathered}
\frac{\mathrm{d} \rho_{\mathrm{S}, i}}{\mathrm{~d} t}=-\beta_{i} \rho_{\mathrm{S}, i} \rho_{\mathrm{I}, i}+\mu \rho_{\mathrm{I}, i}-D_{\mathrm{S}} \rho_{\mathrm{S}, i}+D_{\mathrm{S}} \frac{k_{i}}{\langle k\rangle}\left(\rho-\bar{\rho}_{\mathrm{I}}\right), \\
\frac{\mathrm{d} \rho_{\mathrm{I}, i}}{\mathrm{~d} t}=\beta_{i} \rho_{\mathrm{S}, i} \rho_{\mathrm{I}, i}-\mu \rho_{\mathrm{I}, i}-D_{\mathrm{I}} \rho_{\mathrm{I}, i}+D_{\mathrm{I}} \frac{k_{i}}{\langle k\rangle} \bar{\rho}_{\mathrm{I}} .
\end{gathered}
$$

The endemic equilibrium of the above equations is given by the positive solution of the following equation:

$$
D_{\mathrm{IS}}\left(\rho_{\mathrm{I}, i}^{*}\right)^{2}-\left(\frac{k_{i}}{\langle k\rangle} \gamma\left(\bar{\rho}_{\mathrm{I}}\right)-\frac{\mu+D_{\mathrm{I}}}{\beta_{i}}\right) \rho_{\mathrm{I}, i}^{*}-\frac{D_{\mathrm{I}}}{\beta_{i}} \frac{k_{i}}{\langle k\rangle} \bar{\rho}_{\mathrm{I}}=0,
$$

where $D_{\mathrm{IS}} \equiv D_{\mathrm{I}} / D_{\mathrm{S}}$ and $\gamma\left(\bar{\rho}_{\mathrm{I}}\right) \equiv \rho+\left(D_{\mathrm{IS}}-1\right) \bar{\rho}_{\mathrm{I}}$. See Supplementary Information for the explicit expression of $\rho_{\mathrm{I}, i}^{*}$ as a function of $\bar{\rho}_{\mathrm{I}}$. The self-consistency condition for $\bar{\rho}_{\mathrm{I}}$ yields the following equation:

$$
\bar{\rho}_{\mathrm{I}}=\frac{1}{N}\left(\sum_{i \in H} \rho_{\mathrm{I}, i}^{*}\left(\bar{\rho}_{\mathrm{I}}\right)+\sum_{i \in L} \rho_{\mathrm{I}, i}^{*}\left(\bar{\rho}_{\mathrm{I}}\right)\right),
$$

where $H$ and $L$ denote the sets of indices for the high-risk and low-risk patches, respectively. Denoting the right-hand side of equation (9) by $G\left(\bar{\rho}_{\mathrm{I}}\right)$, the condition for the critical point is given by $G^{\prime}(0)=1$. We can evaluate $G^{\prime}(0)$ as follows:

$$
G^{\prime}(0) \sim(1-p) F\left(\beta_{\mathrm{H}}\right)+p F\left(\beta_{\mathrm{L}}\right),
$$

where $F(\beta)$ is defined in equation (2). By solving $G^{\prime}(0)=1$ with respect to $p$, we obtain the formula for the critical low-risk patch ratio given by equation (1). When inequality (3) does not hold, $F\left(\beta_{\mathrm{H}}\right)$ diverges, and hence, the critical value $p_{c}$ goes to 1. See Supplementary Information for the analysis of the case with limited transmission.

1. Gushulak, B. D. \& MacPherson, D. W. The basic principles of migration health: population mobility and gaps in disease prevalence. Emerg. Themes. Epidemiol 3 , 3 (2006).

2. MacPherson, D. W., Gushulak, B. D. \& Macdonald, L. Health and foreign policy: influences of migration and population mobility. Bull. World Health Organ. 85, 200-206 (2007)

3. United States Centers for Disease Control and Prevention. A CDC Framework for Preventing Infectious Diseases. Available at http://www.cdc.gov/oid/framework. html (last accessed January 10 2013).

4. Hitchcock, P., Chamberlain, A., Van Wagoner, M., Inglesby, T. V. \& O’Toole, T. Challenges to global surveillance and response to infectious disease outbreaks of international importance. Biosecur. Bioterror. 5, 206-227 (2007).

5. Global Outbreak Alert \& Response Network. Available at http://www.who.int/csr/ outbreaknetwork/en/ (last accessed February 2 2014).

6. Hethcote, H. W. An immunization model for a heterogeneous population. Theor. Popul. Biol. 14, 338-349 (1978).

7. Sattenspiel, L. \& Dietz, K. A structured epidemic model incorporating geographic mobility among regions. Math. Biosci. 128, 71-91 (1995).

8. Arino, J. \& van den Drissche. A multi-city epidemics model. Math. Popul. Stud. 10, 175-193 (2003)

9. Keeling, M. J. \& Rohani, P. Modeling Infectious Diseases in Humans and Animals (Princeton University Press, Princeton and Oxford, 2008).

10. Barrat, A., Barthélemy, M., Pastor-Satorras, R. \& Vespignani, A. The architecture of complex weighted networks. Proc. Natl. Acad. Sci. 101, 3747-3752 (2004). 
11. Guimerà, R., Mossa, S., Turtschi, A. \& Amaral, L. A. N. The worldwide air transportation network: Anomalous centrality, community structure, and cities' global roles. Proc. Natl. Acad. Sci. 102, 7794-7799 (2005).

12. Colizza, V., Barrat, A., Barthélemy, M. \& Vespignani, A. The role of the airline transportation network in the prediction and predictability of global epidemics. Proc. Natl. Acad. Sci. 103, 2015-2020 (2006).

13. Sen, P., Dasgupta, S., Chatterjee, A., Sreeram, P. A., Mukherjee, G. \& Manna, S. S. Small-world properties of the Indian railway network. Phys. Rev. E 67, 036106 (2003).

14. Merler, S. \& Ajelli, M. The role of population heterogeneity and human mobility in the spread of pandemic influenza. Proc. Roy. Soc. B 277, 557-565 (2010).

15. Balcan, D., Colizza, V., Gonçalves, B., Hu, H., Ramasco, J. J. \& Vespignani, A. Multiscale mobility networks and the spatial spreading of infectious diseases. Proc. Natl. Acad. Sci. USA 106, 21484-21489 (2009).

16. Balcan, D. \& Vespignani, A. Phase transitions in contagion processes mediated by recurrent mobility patterns. Nat. Phys. 7, 581-586 (2011).

17. Hufnagel, L., Brockmann, D. \& Geisel, T. Forecast and control of epidemics in a globalized world. Proc. Nat. Acad. Sci. 101, 15124-15129 (2004).

18. Watts, D. J., Muhamad, R., Medina, D. C. \& Dodds, P. S. Multiscale, resurgent epidemics in a hierarchical metapopulation model. Proc. Natl. Acad. Sci. USA 102, 11157-11162 (2005).

19. Colizza, V., Pastor-Satorras, R. \& Vespignani, A. Reaction-diffusion processes and metapopulation models in heterogeneous networks. Nat. Phys. 3, 276-282 (2007).

20. Colizza, V. \& Vespignani, A. Invasion threshold in heterogeneous metapopulation networks. Phys. Rev. Lett. 99, 148701 (2007).

21. Colizza, V., Barrat, A., Barthélemy, M., Valleron, A.-J. \& Vespignani, A. Modeling the worldwide spread of pandemic influenza: Baseline case and containment interventions. PLoS Med. 4, e13 (2007).

22. Barrat, A., Barthélemy, M. \& Vespignani, A. Dynamical Processes on Complex Networks (Cambridge University Press, Cambridge, 2008).

23. Colizza, V. \& Vespignani, A. Epidemic modeling in metapopulation systems with heterogeneous coupling pattern: Theory and simulations. J. Theor. Biol. 251, 450-467 (2008).

24. Saldaña, J. Continuous-time formulation of reaction-diffusion processes on heterogeneous metapopulations. Phys. Rev. E 78, 012902 (2008).

25. Juher, D., Ripoll, J. \& Saldaña, J. Analysis and Monte Carlo simulations of a model for the spread of infectious diseases in heterogeneous metapopulations. Phys. Rev. E 80, 041920 (2009).

26. Masuda, N. Effects of diffusion rates on epidemic spreads in metapopulation networks. New J. Phys. 12, 093009 (2010).

27. Belik, V., Geisel, T. \& Brockmann, D. Natural human mobility patterns and spatial spread of infectious diseases. Phys. Rev. X 1, 011001 (2011).

28. Meloni, S., Perra, N., Arenas, A., Gómez, S., Moreno, Y. \& Vespignani, A. Modeling human mobility responses to the large-scale spreading of infectious diseases. Sci. Rep. 1, 62 (2011).

29. Wang, B., Cao, L., Suzuki, H. \& Aihara, K. Safety-information-driven human mobility patterns with metapopulation epidemic dynamics. Sci. Rep. 2, 887 (2012).

30. Allen, L. J. S., Bolker, B. M., Lou, Y. \& Nevai, A. L. Asymptotic profiles of the steady states for an SIS epidemic patch model. SIAM J. Appl. Math. 67, 1283-1309 (2007).

31. Callaway, D. S., Newman, M. E. J., Strogatz, S. H. \& Watts, D. J. Network robustness and fragility: percolation on random graphs. Phys. Rev. Lett. 85, 5468-5471 (2000)

32. Cohen, R., Erez, K., ben-Avraham, D. \& Havlin, S. Breakdown of the Internet under intentional attack. Phys. Rev. Lett. 86, 3682-3685 (2001).
33. Pastor-Satorras, R. \& Vespignani, A. Immunization of complex networks. Phys. Rev. E 65, 036104 (2002).

34. Holme, P., Kim, B. J., Yoon, C. N. \& Han, S. K. Attack vulnerability of complex networks. Phys. Rev. Lett. 65, 056109 (2002).

35. Cohen, R., Havlin, S. \& ben-Avraham, D. Efficient immunization strategies for computer networks and populations. Phys. Rev. Lett. 91, 247901 (2003).

36. Anderson, R. M. \& May, R. M. Infectious Diseases of Humans: Dynamics and Control (Oxford University Press, Oxford, 1991).

37. Murray, J. D. Mathematical Biology: I. An Introduction (Springer, Berlin, 2002)

38. Erdös, P. \& Rényi, A. On the evolution of random graphs. Publications of the Mathematical Institute of the Hungarian Academy of Sciences 5, 17-61 (1960).

39. Barabási, A. L. \& Albert, R. Emergence of scaling in random networks. Science 286, 509-512 (1999).

40. Pastor-Satorras, R. \& Vespignani, A. Epidemic spreading in scale-free networks. Phys. Rev. Lett. 86, 3200-3203 (2001).

41. Dorogovtsev, S. N., Goltsev, A. V. \& Mendes, J. F. F. Critical phenomena in complex networks. Rev. Mod. Phys. 80, 1275-1335 (2008).

42. Tanaka, G., Morino, K. \& Aihara, K. Dynamical robustness in complex networks: the crucial role of low-degree nodes. Sci. Rep. 2, 232 (2012).

43. Freeman, L. C. Centrality in social networks: Conceptual clarification. Social Networks 1, 215-239 (1979).

44. Newman, M. E. J. Networks: An Introduction (Oxford University Press, New York, 2010)

\section{Acknowledgments}

The authors thank Y. Shimada for technical assistance and B. Wang for valuable comments This research was partially supported by JSPS KAKENHI Grant No. 24700222 (GT) and the Aihara Innovative Mathematical Modelling Project, the Japan Society for the Promotion of Science (JSPS) through the "Funding Program for World-Leading Innovative R\&D on Science and Technology (FIRST Program)," initiated by the Council for Science and Technology Policy (CSTP) (GT, CU, and KA).

\section{Author contributions}

G.T. conceived the research. G.T. and C.U. designed the research, carried out the numerical experiments, and worked out the theoretical analyses. G.T., C.U. and K.A. wrote, reviewed, and approved the manuscript.

\section{Additional information}

Supplementary information accompanies this paper at http://www.nature.com/ scientificreports

Competing financial interests: The authors declare no competing financial interests.

How to cite this article: Tanaka, G., Urabe, C. \& Aihara, K. Random and Targeted Interventions for Epidemic Control in Metapopulation Models. Sci. Rep. 4, 5522; DOI:10.1038/srep05522 (2014).

This work is licensed under a Creative Commons Attribution-NonCommercialShareAlike 4.0 International License. The images or other third party material in this article are included in the article's Creative Commons license, unless indicated otherwise in the credit line; if the material is not included under the Creative Commons license, users will need to obtain permission from the license holder in order to reproduce the material. To view a copy of this license, visit http:// creativecommons.org/licenses/by-nc-sa/4.0/ 